Pacific Journal of Mathematics

CONCERNING KOCH'S THEOREM ON THE EXISTENCE OF

ARCS 


\title{
CONCERNING KOCH'S THEOREM ON THE EXISTENCE OF ARCS
}

\author{
L. E. WARD, JR.
}

A theorem of R. J. Koch asserts that if $X$ is a compact space endowed with a partial order $I$ such that

(i) $\Gamma$ is a closed subset of $X \times X$,

(ii) there exists $0 \in X$ such that $(0, x) \in \Gamma$ for each $x \in X$, and

(iii) for each $x \in X$ the set $L(x)=\{y: y \leqq x\}$ is connected, then each point of $X$ lies in a connected chain containing 0 . In particular, $X$ is arcwise connected. This is a corollary of the theorem: if $X$ is a compact space and $l^{\prime}$ is a partial order satisfying (i), and if $W$ is an open subset of $X$ such that each neighborhood of each point $x$ of $W$ contains a point $y \neq x$ with $(y, x) \in \Gamma$, then each point of $W$ is the supremum of a connected chain which meets $X-W$. A new proof of these results is presented.

The first of these theorems is generalized in several ways. The compactness is relaxed to local compactness and the assumption that each closed chain has a zero. Moreover, the existence of a zero need not be assumed. If the set $E$ of minimal elements is closed, then $E$ is joined by connected chains to all other points of $X$. If the set function $L$ is continuous, then $E$ is necessarily closed.

1. A classical problem of topology is to determine when a space is arcwise connected. Here it will be convenient to adopt the terminology of A. D. Wallace [6] and call a subset $A$ of a space an arc if $A$ is a continuum with exactly two noncutpoints. If $A$ is also separable then it is a real arc.

A few years ago R. J. Koch [4] proved a remarkable theorem of this type. He showed that a compact partially ordered space is arcwise connected if certain natural conditions are imposed on the partial order. It is the purpose of this paper to study Koch's result in detail. His proof, although ingenious, is long and very complicated. Since the theorem is fundamental to the structure theory of partially ordered spaces, and since it has been applied [3, 4, 6] to a variety of problems in topological algebra, it is of some interest to exhibit a shorter and simpler proof. This is done in $\$ 2$. In the later sections, some generalizations of Koch's theorem are obtained.

Received February 13, 1964. Presented to the American Mathematical Society, January 24, 1964. The author gratefully acknowledges the support of the National Science Foundation. 
Perhaps the most celebrated theorem on arcwise connectivity is the assertion that every locally connected and metrizable continuum is real arcwise connected. I suspect the existence of an intimate relationship between Koch's theorem and this result. In the final section of the present paper, this possible relationship is discussed, but I have not been able to resolve the question satisfactorily.

2. A short proof of Koch's theorem. If $\Gamma$ is a partial order on a set $X$, we identify $\Gamma$ with its graph and treat the symbols $x \leqq$ $y, x \Gamma y$ and $(x, y) \in \Gamma$ as synonyms. Recall that a chain of a partially ordered set $(X, \Gamma)$ is a subset $C$ of $X$ such that $a \Gamma b$ or $b \Gamma a$ obtains for each $a$ and $b$ in $C$. We also define

$$
\begin{aligned}
L(a, \Gamma) & =\{x \in X: x \Gamma a\}, \\
M(a, \Gamma) & =\{x \in X: a \Gamma x\},
\end{aligned}
$$

for each $a \in X$. Where no ambiguity may occur we shall write $L(a)$ for $L(a, \Gamma)$ and $M(a)$ for $M(a, \Gamma)$. Moreover, if $A \subset X$ we define

$$
L(A)=\bigcup\{L(x): x \in A\},
$$

and it is convenient to adopt the notation

$$
[x, y]_{\Gamma}=M(x, \Gamma) \cap L(x, \Gamma) .
$$

In case $X$ is a topological space, the partial order $\Gamma$ is continuous provided $\Gamma$ is a closed subset of $X \times X$. When this occurs, $X=$ $(X, \Gamma)$ is called a continuously partially ordered space. It is wellknown [7] that if $X$ is a continuously partially ordered space then the sets $L(x)$ and $M(x)$ are closed, for each $x \in X, X$ is a Hausdorff space, and, if $X$ is compact, it admits a minimal element, i.e., an element having no proper predecessors. A zero of a continuously partially ordered space is an element which precedes every other element. In the compact case, a unique minimal element is necessarily a zero. Finally, we remark that in a compact, continuously partially ordered space, a connected chain joining two distinct points is an arc. An arc which is also a chain will be termed an order arc or a $\Gamma$-arc.

(2.1) THEOREM (Koch). Let $W$ be an open subset of the compact, continuously partially ordered space $X$, and suppose, for each $x \in W$, that each neighborhood of $x$ contains an element $y$ with $y>x$. Then each $x \in W$ is the supremum of an order arc $C$ such that $C-W$ is nonempty.

(2.2) CoRollary. If $X$ is a compact, continuously partially ordered space with zero, 0 , such that $L(x)$ is connected for each $x \in X$, then each $x \in X-\{0\}$ is joined to 0 by an order arc. 
The corollary follows easily from Theorem 2.1 by taking $W=$ $X-\{0\}$ (see [4]). Our proof of Theorem 2.1 is embodied in two main lemmas.

(2.3) Lemma. Let $W$ be an open subset of the compact space $X$. If $X$ admits a partial order satisfying the hypotheses of (2.1), then $X$ admits such a partial order which is minimal.

Proof. Let $\left\{\Gamma_{\alpha}\right\}$ be a maximal nest of partial orders satisfying the hypotheses of (2.1), and let $\Gamma_{0}=\bigcap\left\{\Gamma_{\alpha}\right\}$. It is readily verified that $\Gamma_{0}$ is a continuous partial order on $X$. Let $x \in W$ and let $U$ be a neighborhood of $x$; clearly we may assume that $U \subset W$. Since $X$ is regular, there exists an open set $V$ with $x \in V \subset \bar{V} \subset U$, and since $X$ is normal, there exists an open set $R$ with $X-U \subset R \subset \bar{R} \subset X-\bar{V}$. For each $\alpha$, let $x_{\alpha}$ be a $\Gamma_{\alpha}$-minimal element of $L\left(x, \Gamma_{\alpha}\right) \cap \bar{V}$; then there must exist $y_{a} \neq x_{a}$ such that

$$
y_{\alpha} \in L\left(x_{\alpha}, \Gamma_{\alpha}\right)-\bar{R} \subset L\left(x, \Gamma_{\alpha}\right)-(\bar{R} \cup \bar{V}) .
$$

Since the closed sets $L\left(x, \Gamma_{a}\right)-(R \cup V)$ are nested and nonempty, there exists $y \in L\left(x, \Gamma_{0}\right)-R \cup V$. Thus $(y, x) \in \Gamma_{0}, y \neq x$, and $y \in U$. Therefore $\Gamma_{0}$ satisfied the hypotheses of (2.1) and is minimal.

(2.4) Lemma. Let $W$ be an open subset of the compact space $X$, and suppose $\Gamma$ is a partial order on $X$ which is minimal with respect to satisfying the hypotheses of (2.1). Then every maximal chain of $(X, \Gamma)$ is connected.

Proof. If not then the compactness of $X$ guarantees [7] the existence of elements $a$ and $b$ of $X$ with $(a, b) \in \Gamma, a \neq b$, and

$$
[a, b]_{\Gamma}=\{a\} \cup\{b\} \text {. }
$$

Since $X$ is a Hausdorff space, there are disjoint open sets $U$ and $V$ with $a \in U$ and $b \in V$. Let

$$
F=\left\{(x, y) \in X \times X:[x, y]_{\Gamma}-(U \cup V) \neq 0\right\} .
$$

A routine argument involving the continuity of $\Gamma$ shows that $F$ is closed and hence

$$
\Delta=\Gamma-(U \times V-F)
$$

is also closed. Since $\Gamma$ is reflexive and $U \cap V=0$, one sees that $\Delta$ is reflexive, and the anti-symmetry of $\Gamma$ implies that $\Delta$ has the same property. To see that $\Delta$ is transitive, suppose that $p \Delta q$ and $q \Delta r$ but $(p, r) \in X \times X-4$. Since $p \Gamma r$, it is clear that 


$$
(p, r) \in U \times V-F
$$

and thus $[p, r]_{\Gamma} \subset U \cup V$, so that $q \in U$ or $q \in V$. If $q \in U$ then, since $r \in V$ and $(q, r) \in \Delta$, we infer that $(q, r) \in F$ and consequently

$$
[q, r]_{\Gamma}-(U \cup V) \neq 0 \text {. }
$$

But then

$$
[p, r]_{\Gamma}-(U \cup V) \neq 0,
$$

i.e., $(p, r) \in F$, a contradiction. A similar contradiction ensues if $q \in V$ and therefore $\Delta$ is transitive.

Now let $x \in W$ and let $N$ be a neighborhood of $x$. If $x \in X-V$ then $L(x, \Delta)=L(x, \Gamma)$ and hence there exists $y \in N, y \neq x$, with $y \Delta x$. If $x \in V$ then

$$
L(x, \Delta) \cap V=L(x, \Gamma) \cap V
$$

and hence the desired $y$ exists in $N \cap V$. Therefore $\Delta$ satisfies the hypotheses of (2.1), contrary to the minimality of $\Gamma$.

Proof of Theorem (2.1). In view of Lemma 2.3 we may assume that $\Gamma$ is minimal, for any $\Gamma$-arc will be an order arc with respect to a partial order which contains $\Gamma$. Let $x \in W$ and let $D$ be a maximal chain of $X$ such that $x \in D$. By Lemma 2.4, $D$ is an order arc, and by hypothesis, $C=D \cap L(x)$ is nondegenerate and hence $C$ is also an order arc. Since $X$ is compact, $C$ has a least element which cannot lie in $W$.

It should be noted that the chief applications to topological algebra arise from Theorem 2.1. From a purely topological point of view, hewever, Corollary 2.2 is the more interesting, and it is this result which we shall generalize in several ways.

3. A lemma on quotient spaces. If $X$ is a space and $F$ is a closed subset of $X$, we denote by $X / F$ the quotient space which is obtained when $F$ is identified with a point.

(3.1) Lemma. Let $(X, \Gamma)$ be a continuously partially ordered space and let $F$ be a compact subset of $X$ such that $F=L(F)$. Then $X / F$ is a continuously partially ordered space. If, for each $x \in X$, it follows that $L(x, \Gamma)$ meets $F$, then $F$ is a zero for $X / F$. Finally, if $X$ is compact and, for each $x \in X$, each component of $L(x, \Gamma)$ meets $F$, then $X / F$ satisfies the hypotheses of Corollary 2.2 and hence each point of $X / F-\{F)$ is joined to $F$ by an order arc of $X / F$. 
Proof. Define the relation $\Delta$ on $X / F$ by $p \Delta q$ provided $p, q \in X-F$ and $p \Gamma q$, or $p=F$ and $L(q, \Gamma)$ meets $F$. It is clear that $\Delta$ is a partial order, and the proof that $\Delta$ is continuous is routine except for the verification of the fact that if $(F, q) \notin \Delta$, then there are open sets $U$ and $V$ such that $q \in U, F \subset V$ and $L(U, \Gamma)$ and $V$ are disjoint. To see this we note that since $\Gamma$ is continuous and $L(q, \Gamma)$ and $F$ are disjoint there exist, for each $t \in F$, open sets $U_{t}$ and $V_{t}$ such that $q \in U_{t}, t \in V_{t}$ and $L\left(U_{t}, \Gamma\right)$ and $V_{t}$ are disjoint. Since $F$ is compact, a familiar argument shows that the desired sets $U$ and $V$ exist. That $F$ is a zero if each $L(x, I)$ meets $F$ is obvious. If $X$ is compact then so is $X / F$, and if each component $K_{\alpha}$ of $L(x, \Gamma)$ meets $F$, then

$$
L(x, \Delta)=\{F\} \cup \bigcup\left\{K_{\infty}-F\right\}
$$

is also connected.

(3.2) COROLLARY. If $X$ is a compact and continuously partially ordered space, if $F$ is a closed subset of $X$ such that $F=L(F)$ and if, for each $x \in X$, each component of $L(x)$ meets $F$, then, for each $x \in X-F$, there exists $y<x$ such that $y$ and $x$ are joined by an order arc in $X$.

Proof. If $x \in X-F$, then, in $X / F$, there exists an order arc $A_{x}$ joining $F$ and $x$. Let $y \in A_{x}-\{x\} \cup\{F\}$; then $y<x$ and an order arc joins $y$ and $x$ in $X / F$. Since this arc is disjoint from $F$, it remains an order arc in $X$.

In the following sections we shall also require a simple lemma about compact partially ordered spaces.

(3.3) LemMa. If $A$ is a closed subset of a compact, continuously partially ordered space, then $L(A)$ is a closed set.

Proof. Let $\Gamma$ denote the graph of the partial order. Choquet [2] first observed ${ }^{1}$ that in a continuously partially ordered space the set functions $L$ and $M$ are upper semi-continuous. Therefore, if $x \notin L(A)$, there is an open set $U$ with $x \in U$ such that $M(t) \cap A=0$ for each $t \in U$. Therefore $U \cap L(A)=0$, so that $L(A)$ is closed.

4. The locally compact case. Very simple examples exist to show that Koch's theorem fails if $X$ is assumed only to be locally compact. For later reference we describe one of these.

(4.1) ExAMPLE. There exists a locally compact and continuously partially ordered space $Y$ with zero, 0 , such that $L(x)$ is connected,

1 I am indebted to the referee for this reference. 
for each $x \in Y$, but certain elements of $Y-\{0\}$ are not joined to 0 by an arc.

In the Cartesian plane let $A_{-1}$ denote the closed line segment whose endpoints are $(0,0)$ and $(1,0), A_{0}$ is the closed line segment whose endpoints are $(1,0)$ and $(1,1)$, and, for each $n=1,2, \cdots, A_{n}$ is the closed line segment whose endpoints are $\left(1-2^{n}, 0\right)$ and $\left(1-2^{n}, 1\right)$. Let

$$
X=\bigcup_{n=-1}^{\infty}\left\{A_{n}\right\}
$$

In the relative topology $X$ is a compact space. Give $X$ the coordinatewise partial order, i.e., $(a, b) \leqq(c, d)$ if and only if $a \leqq c$ and $b \leqq d$. Then it is easy to see that $X$ satisfies the hypotheses of Theorem 2.1, with the origin for zero.

Now let $S$ be a closed segment of $A_{0}$ which does not contain $(1,1)$, and let $Y=X-S$. Then $Y$ is a locally compact space which otherwise satisfies all the hypotheses of Theorem 2.1, but no arc joins 0 to $(1,1)$.

The space $Y$ is even a topological semi-lattice. The author and L. W. Anderson [1] have shown that if a connected and locally compact topological lattice has a zero, then each point is connected by an order arc to zero, and, under suitable auxiliary hypotheses, the same is true of locally compact semi-lattices, but our methods depended very strongly on the lattice structure.

With no additional hypotheses at all, however, some results can be obtained in the locally compact case, using Lemma 3.1 and Corollary 3.2 .

(4.2) THEOREM. Let $X$ be a continuously partially ordered space, let $p \in X$, and suppose $p$ admits a compact neighborhood $N$ which contains no minimal elements of $X$. If $L(x)$ is connected, for each $x \in N$, then there exists $q \in L(p)-\{p\}$ such that $q$ and $p$ lie in an order arc.

Proof. Let $B$ denote the boundary of $N$ and define

$$
F=L(L(p) \cap B) \cap N .
$$

We assert that $L(p) \cap B$ is not empty, for otherwise the connectivity of $L(p)$ insures that $L(p) \subset N$; but then $L(p)$ is compact and hence contains a minimal element of $X$. But, by hypothesis, $N$ contains no minimal elements of $X$. Moreover, since $p \in L(p)-B$, it follows that $p \in L(p) \cap(N-F)$. By Lemma 3.3, $F$ is a closed subset of $L(p) \cap N$. If $x \in L(p) \cap(N-F)$ then the connectivity of $L(x)$ guarantees that 
each component of $L(x) \cap N$ meets $F$. Therefore, the space $L(p) \cap N$ satisfies the hypotheses of Corollary 3.2, and the theorem follows.

Referring to the space $Y$ of Example 4.1, the point $(1,1)$ can certainly be joined by an order arc to a point $(1,1-\varepsilon)<(1,1)$. In order to continue this arc on to 0 it is necessary to add some further hypothesis such as is contained in our next result.

(4.3) THEOREM. Let $X$ be a locally compact, continuously partially ordered space with zero, 0 , and suppose $L(x)$ is connected, for each $x \in X$. If each closed chain of $X$ has a zero, then each $x \in X-$ $\{0\}$ is joined to 0 by an order arc.

Proof. If $x \in X-\{0\}$, then Theorem 4.2 assures us that $x$ is the supremum of a nontrivial connected chain. Let $C$ be a maximal such chain; by hypothesis, $z(C)$, the zero of $C$, exists. If $z(C) \neq 0$, then another application of Theorem 4.2 produces a nontrivial connected chain $D$, of which $z(C)$ is the supremum. But the chain $C \cup D$ is connected and thus contradicts the maximality of $C$. Thus $C$ is an order arc joining $x$ to 0 .

We note that Theorem 4.3 truly generalizes Corollary 2.2 because, in the compact case, every closed chain has a zero.

Problem. Does Theorem 4.3 remain true if the hypothesis that each closed chain has a zero is weakened to "each chain has an infimum"?

5. Partially ordered spaces without zero. Let $K$ be a continuum which contains no arc. Select $x_{1} \in K$ and define $x \leqq y$ if and only if $y=x_{1}$ or $y=x$. With respect to this relation $K$ is a compact continuously partially ordered space in which each set $L(x)$ is connected but in which there are no arcs. Thus we cannot infer the existence of order arcs without some restrictions on the set of minimal elements, but the hypothesis of Corollary 2.2 that there is only one minimal element is unduly restrictive.

(5.1) THEOREM. Let $X$ be a compact, continuously partially ordered space in which $L(x)$ is connected, for each $x \in X$. Let $E$ denote the set of minimal elements of $X$, and suppose, for each $x \in X-E$, that $x \in X-C l(L(x) \cap E)$. Then each $x \in X-E$ is joined by an order arc to some element of $E$.

Proof. Let $x \in X-E$; since $L(x)$ is also a compact, continuously partially ordered space, $L(x) \cap E$ is not empty. Let

$$
E_{x}=L(C l(L(x) \cap E))
$$


and note that $x \in L(x)-E_{x}$ and, by Lemma 3.3 , that $E_{x}$ is closed. By Corollary 3.2, $x$ is the supremum of a nondegenerate connected chain. The proof now follows that of Theorem 4.3. If $C$ is a maximal connected chain such that $x=\sup C$, then by compactness $C$ has a zero which, by maximality, is a member of $E$.

(5.2) COROLlaRY. Let $X$ be a locally compact, continuously partially ordered space in which each closed chain has a zero, and in which, for each $x \in X-E$, it follows that $x \in X-C l(L(x) \cap E)$, where $E$ denotes the set of minimal elements of $X$. If $L(x)$ is connected, for each $x \in X$, then each $x \in X-E$ is joined by an order arc to some element of $E$.

Proof. If $x \in X-E$ then by Theorem $4.2, x$ is the supremum of some nondegenerate connected chain. If $C$ is a maximal chain with this property, then $C$ is closed and, by maximality, its zero is an element of $E$.

(5.3) COROLlaRY. Let $X$ be a locally compact, continuously partially ordered space in which each closed chain has a zero, and in which the set $E$ of minimal elements is closed. If $L(x)$ is connected, for each $x \in X$, then each $x \in X-E$ is joined by an order arc to some element of $E$.

Some authors have called a partial order on a space "continuous" if the set-valued mapping $L$ is continuous in the following sense: that each set $L(x)$ is closed and, if $U$ and $V$ are open sets such that $L(x) \subset U$ and $L(x)$ meets $V$, then there exists an open set $W$ containing $x$ such that, if $y \in W$, then $L(y) \subset U$ and $L(y)$ meets $V$. If a partial order satisfies this condition, let us say that the space is an $L$-continuous partially ordered space. It is a simple exercise to verify that $L$-continuity of a partial order implies continuity. (See Choquet [2].)

(5.4) THEOREM. If $X$ is an L-continuous partially ordered space, then the set $E$ of minimal elements of $X$ is closed.

Proof. If $x \in X-E$ then there exists $p<x$ and hence, if $U$ is a neighborhood of $p, L(x) \cap U$ is not empty. We may select $U$ such that $x \in X-\bar{U}$. By $L$-continuity, there exists an open set $W$ such that $x \in W \subset X-\bar{U}$ and, for each $t \in W, L(t) \cap U$ is not empty. In particular, $L(t)$ is nondegenerate and hence $W \cap E$ is empty.

(5.5) CoRollary. If $X$ is a locally compact, L-continuous partially ordered space in which each closed chain has a zero, and if 
$L(x)$ is connected, for each $x \in X$, then each non-minimal element of $X$ is joined by an order arc to some minimal element of $X$.

6. Concluding remarks. We return to consideration of the theorem that a locally connected, metrizable continuum is real arcwise connected. The problem we wish to raise may be put in this way: Does Koch's theorem imply the arcwise connectivity of such continua? Since Mardešic has shown [5] that the natural analog of this result fails in the nonmetrizable case, metrizability (or some slightly weaker condition) must certainly be assumed. Now it can be shown that any locally connected continuum admits a nontrivial quasi-order (i.e., a reflexive, transitive relation) which is continuous, has a zero, and is such that each set $L(x)$ is connected. By an argument similar to that of Lemma 2.3 one can find a minimal quasi-order with the same properties. If, under suitable conditions, this minimal quasi-order is found to be a partial order, then arcwise connectivity would follow from Corollary 2.2.

\section{REFERENCES}

1. L. W. Anderson and L. E. Ward, Jr., A structure theorem for topological lattices, Proc. Glasgow Math. Assoc. 5 (1961), 1-3.

2. G. Choquet, Convergences, Annales Grenoble Univ., Sec. des Sci., Math. et Phys. 23 (1947), 58-112.

3. R. P. Hunter, Note on arcs in semigroups, Fund. Math. 49 (1961), 233-245.

4. R. J. Koch, Arcs in partially ordered spaces, Pacific J. Math. 9 (1959), 723-728.

5. S. Mardešic, On the Hahn-Mazurkiewicz theorem in non-metric spaces, Proc. Amer. Math. Soc. 11 (1960), 929-937.

6. A. D. Wallace, Relations on topological spaces, Proceedings of the Symposium on General Topology, Prague, (1961), 356-360.

7. L. E. Ward, Jr. Partially ordered topological spaces, Proc. Amer. Math. Soc. 5 (1954), 144-161. 



\section{PACIFIC JOURNAL OF MATHEMATICS}

\section{EDITORS}

H. Samelson

Stanford University

Stanford, California

R. M. Blumenthal

University of Washington

Seattle, Washington 98105
J. Dugundu

University of Southern California Los Angeles, California 90007

*Richard Arens

University of California

Los Angeles, California 90024

\section{ASSOCIATE EDITORS}
E. F. BECKENBACH
B. H. NeumanN
F. WOLF
K. YOSIDA

\section{SUPPORTING INSTITUTIONS}

UNIVERSITY OF BRITISH COLUMBIA CALIFORNIA INSTITUTE OF TECHNOLOGY UNIVERSITY OF CALIFORNIA MONTANA STATE UNIVERSITY

UNIVERSITY OF NEVADA

NEW MEXICO STATE UNIVERSITY

OREGON STATE UNIVERSITY

UNIVERSITY OF OREGON

OSAKA UNIVERSITY

UNIVERSITY OF SOUTHERN CALIFORNIA
STANFORD UNIVERSITY

UNIVERSITY OF TOKYO

UNIVERSITY OF UTAH

WASHINGTON STATE UNIVERSITY

UNIVERSITY OF WASHINGTON

AMERICAN MATHEMATICAL SOCIETY CALIFORNIA RESEARCH CORPORATION SPACE TECHNOLOGY LABORATORIES NAVAL ORDNANCE TEST STATION 


\section{Pacific Journal of Mathematics}

\section{Vol. 15, No. $1 \quad$ September, 1965}

Donald Charles Benson, Unimodular solutions of infinite systems of linear

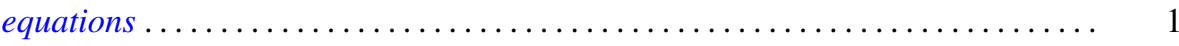

Richard Earl Block, Transitive groups of collineations on certain designs . . . . . . 13

Barry William Boehm, Existence of best rational Tchebycheff approximations .... . 19

Joseph Patrick Brannen, A note on Hausdorff's summation methods . . . . . . . . . . 29

Dennison Robert Brown, Topological semilattices on the two-cell ............ 35

Peter Southcott Bullen, Some inequalities for symmetric means . . . . . . . . . . 47

David Geoffrey Cantor, On arithmetic properties of coefficients of rational

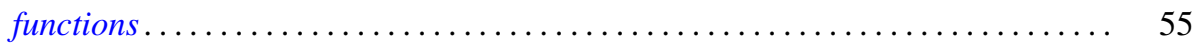

Luther Elic Claborn, Dedekind domains and rings of quotients . . . . . . . . . 59

Allan Clark, Homotopy commutativity and the Moore spectral sequence ........ 65

Allen Devinatz, The asymptotic nature of the solutions of certain linear systems of

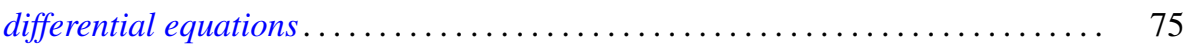

Robert E. Edwards, Approximation by convolutions ................... 85

Theodore William Gamelin, Decomposition theorems for Fredholm operators . . . . . 97

Edmond E. Granirer, On the invariant mean on topological semigroups and on

topological groups .................................. 107

Noel Justin Hicks, Closed vector fields . . . . . . . . . . . . . . . . . . . 141

Charles Ray Hobby and Ronald Pyke, Doubly stochastic operators obtained from

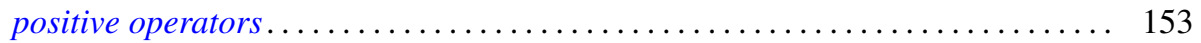

Robert Franklin Jolly, Concerning periodic subadditive functions . . . . . . . . . 159

Tosio Kato, Wave operators and unitary equivalence . . . . . . . . . . . . . . 171

Paul Katz and Ernst Gabor Straus, Infinite sums in algebraic structures . . . . . . . 181

Herbert Frederick Kreimer, Jr., On an extension of the Picard-Vessiot theory ...... 191

Radha Govinda Laha and Eugene Lukacs, On a linear form whose distribution is

identical with that of a monomial ......................... 207

Donald A. Ludwig, Singularities of superpositions of distributions . . . . . . . . . 215

Albert W. Marshall and Ingram Olkin, Norms and inequalities for condition

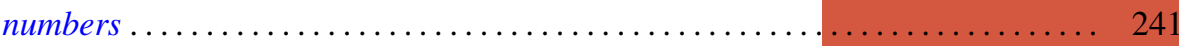

Horace Yomishi Mochizuki, Finitistic global dimension for rings . . . . . . . . . . 249

Robert Harvey Oehmke and Reuben Sandler, The collineation groups of division

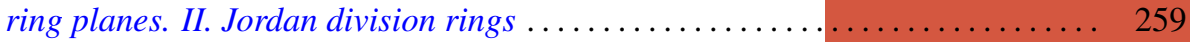

George H. Orland, On non-convex polyhedral surfaces in $E^{3} \ldots \ldots \ldots \ldots \ldots \ldots \ldots 267$

Theodore G. Ostrom, Collineation groups of semi-translation planes . . . . . . . . 273

Arthur Argyle Sagle, On anti-commutative algebras and general Lie triple

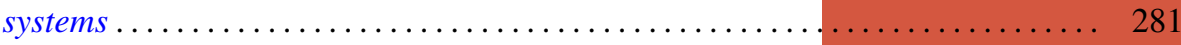

Laurent Siebenmann, A characterization of free projective planes . . . . . . . . . 293

Edward Silverman, Simple areas.................................. 299

James McLean Sloss, Chebyshev approximation to zero .................. 305

Robert S. Strichartz, Isometric isomorphisms of measure algebras . . . . . . . . . 315

Richard Joseph Turyn, Character sums and difference sets . . . . . . . . . . . . 319

L. E. Ward, Concerning Koch's theorem on the existence of arcs . . . . . . . . . . 347

Israel Zuckerman, A new measure of a partial differential field extension ......... 357 